\title{
Strong correlations between structural order and passive state at water- copper oxide interfaces
}

\author{
Badri Narayanan, ${ }^{1}$ Sanket A. Deshmukh, ${ }^{1}$ Subramanian K. R. S. Sankaranarayanan ${ }^{{ }^{*}}$ and \\ Shriram Ramanathan ${ }^{2}$ \\ ${ }^{1}$ Center for Nanoscale Materials, Argonne National Laboratory, Argonne, Illinois 60439, \\ USA \\ ${ }^{2}$ School of Engineering and Applied Sciences, Harvard University, Cambridge, \\ Massachusetts 02138, USA \\ * Email for correspondence: skrssank@anl.gov
}

\begin{abstract}
A fundamental understanding of coupled electrochemical processes including metal dissolution, structural evolution and solvation dynamics at the atomic level is of interest to corrosion research and electrochemistry in general. Using molecular dynamics (MD) simulations based on a reactive force field (ReaxFF), we evaluate the impact of nonstoichiometry in a model system of copper oxide passive films on the local fluctuation of the chloride ion density and structure and dynamics of interfacial water layers. We investigate (a) the interplay of oxygen content in the passive oxide film and the solvation dynamics of halide ions in the aqueous interfacial layers during breakdown of the oxide film, and (b) their combined effects on the dissolution kinetics of copper and adsorption of chloride ions on the copper-oxide surface. We demonstrate that the solvation behavior, particularly near the oxide/aqueous medium interface, is strongly correlated with the interfacial chloride ion concentration, which in turn is influenced by the oxygen stoichiometry in the passive oxide. Residence probability and hydrogen-bond correlations show that water present in the aqueous media forms ordered layers on oxide films with high oxygen content; and as the oxygen content is reduced, this order gets disrupted due to increased chloride ion adsorption. Interfacial molecular order is therefore strongly correlated with stoichiometry of the passive oxide film.
\end{abstract}




\section{Introduction}

Open questions pertaining to atomistic understanding of initiation of corrosion as well as nanoscale mechanisms of breakdown of the passive metal oxide films include probing the (a) role of water and solute ions on the oxide structure, composition, and reactivity at defective surface sites, (b) interfacial distribution of cations and anions in the oxide layer as well as solution, (c) charge transfer dynamics at the passive oxidewater interface, and (d) mechanisms of ion adsorption and transport, and their effect on corrosion resistance of the passive oxide layer [1-6]. Additionally, there are several important questions pertaining to solvation dynamics near electrochemical interfaces, ionic transport across electrochemical double layers as well as interfacial molecular order in a typical electrochemical environment that are not yet well understood [7-9].

Several macroscopic theories as well as simulation and experimental reports exist in the literature, which attempt to explain the mechanistic sequence of events leading to corrosion initiation and passive oxide breakdown. The atomistic origin of these events and nanoscale pitting mechanism remain an active area of study $[10,11]$. Most corrosion models at continuum or atomistic scale focus on either ion adsorption or transport through passive oxides [12-14]. While the continuum scale kinetic models such as the point defect models [15-17] can capture the description of the growth and breakdown of the passive oxide film, the atomistic details involving bond formation and bond breakage in a reactive aqueous environment cannot be elucidated. There have been a few classical molecular dynamics simulations $[5,6,9,18]$ and first-principles studies [19-21] in the past to understand the dynamics and energetics of chloride ion adsorption onto the surface of passive oxides. These studies including our recent study [5] have 
focused on the effect of chloride ion concentration and ambient temperature on passive oxide breakdown. A systematic evaluation of the role of oxygen non-stoichiometry on passive oxide breakdown and the interplay between the non-stoichiometric oxide composition and solvation dynamics of interfacial aqueous layers is the primary focus of this study.

The ordering of water molecules into "ice-like" layers near the interface between water (or aqueous solutions) and several oxides e.g., $\mathrm{RuO}_{2}$ [22], mica [23], among various other minerals [24-26] have been investigated using specular reflectivity measurements, spectroscopic and electron diffraction experiments [27]. These reports indicate that water molecules near the oxide/water interface undergo spatial ordering to form hydration layers owing to either (a) completion of coordination shells of surface cations, or (b) interactions between surface oxygen atoms in the oxide and the water molecules [27]. Such an ordering can occur over a few layers (e.g., monolayer at $\mathrm{RuO}_{2}$ /water [22], 2-3 layers at $\mathrm{MgO} /$ water interfaces [28, 29]); in extreme cases, weak ordering could exist over larger length scales $\sim 1 \mathrm{~nm}$ from the interface, e.g., muscovite mica [23, 27]. Apart from these experimental reports, researchers have also probed this ordering phenomenon using atomistic simulations [28-32]. In terms of corrosive aqueous environments, the presence of halide anions can profoundly alter the structure and dynamics of interfacial water layers $[18,33]$. While the solvation characteristics of interfacial layers are expected to play an important role in corrosion initiation, they have received much less attention to date [7, 33]. Recently, we have shown that ionic solvation of water near passive oxides could also play an important role in influencing halide ion adsorption and transport [18]. Chloride anions beyond a certain threshold concentration 
can significantly alter the dynamics of re-arrangement of hydrogen bonding (HB) network and local interfacial ordering near a passive oxide such as $\mathrm{MgO}$. We note that the solvated ions have important functional roles in screening or moving charge, and can thus potentially influence the kinetics of breaking and structural relaxation of ion-water and water-water HB in these solutions. These, in turn, greatly influence the mobility of both the ions and water molecules. In the case of interfacial water, it would be fair to state that the physical picture of solvation dynamics i.e. HB network connectivity and its response to aggressive anions is still emerging [34].

In this work, we use a model system consisting of copper oxide thin film on copper interacting with chloride ions in aqueous media to gain a comprehensive understanding of representative processes that occur at the interface of passive film and aqueous media. We utilize reactive molecular dynamics simulations and investigate the temporal evolution of copper oxides and surface properties of the passive oxides in an aqueous media containing halide ions. We chose an alkaline environment with excess hydroxyl ions since it is known to show a more aggressive corrosion behavior that can be captured within MD timescales [5, 6]. We evaluate the role of oxygen non-stoichiometry in the passive oxide and focus on the early stages of chemical failure. In addition, we demonstrate how oxide non-stoichiometry profoundly affects the solvation dynamics of water proximal to a passive oxide. We elucidate the atomistic level correlation between the oxide non-stoichiometry, concentration of chloride ions as well as the molecular order, diffusional characteristics and HB characteristics of interfacial water. To the best of our knowledge, this work represents the first large scale reactive atomistic scale simulations 
that provides fundamental insights into the interplay between the oxide non-stoichiometry and interfacial solvation behavior during aqueous corrosion of copper oxides.

\section{Computational details}

We performed reactive molecular dynamics (MD) simulations in the canonical ensemble (NVT) to systematically investigate the effect of surface oxide stoichiometry on the corrosion behavior of copper in an aqueous environment. The interactions between the $\mathrm{Cu}, \mathrm{O}, \mathrm{H}$, and $\mathrm{Cl}$ atoms were modeled by a general bond-order based reactive force field (ReaxFF), which is known to describe accurately the formation and dissociation of chemical bonds [35-38]. In the framework of ReaxFF, the total energy of the system is composed of several contributions arising from connectivity-dependent interactions such as, bond stretching, angle bending, dihedral twisting, lone pairs, and under/over coordination. All these short-range interactions are functions of bond orders that are derived from instantaneous interatomic distances; the bond order for each pair of atoms depends strongly on its local environment, and thereby, accounts for many-body effects [35-37]. Additionally, long-range interactions (i.e., Coulomb and van der Waals) are computed for every atom-pair regardless of their connectivity. The temporal evolution of atomic charges is evaluated using electronegativity equalization method [39]. This approach enables ReaxFF to describe metallic, covalent, and ionic systems equally well [35-38]. ReaxFF has been successfully employed to study a wide range of materials, including metals [40, 41], metal oxides [41-43], mixed ceramic oxides [44, 45], hydrocarbons [35, 37], chlorides and aqueous solutions [5, 6, 38]. The ReaxFF parameters used in this study were taken from Ref. [38]. These parameters were found to accurately predict formation energies of $\mathrm{Cu}$ oxides [41] and $\mathrm{Cu}-\mathrm{Cl}$ clusters [38]; in 
previous works, we have employed these parameters to identify atomic-scale mechanisms of aqueous corrosion of $\mathrm{Cu}$, and investigate $\mathrm{Cl}^{-}$ion interaction with copper oxide surfaces $[5,6]$.

All the MD simulations were performed using the classical MD simulation package LAMMPS [46] with a timestep of 0.5 fs. Following our earlier works [5, 6], we employed a slightly larger timestep than that usually used for ReaxFF MD study of hydrocarbon/water systems (0.25 fs; e.g., Ref. [38]) to make longer MD runs (2 ns) tractable whilst capturing the dynamics accurately. In our MD simulations, using the larger timestep, the fluctuations in the total energy of the equilibrated configurations were found to be within $0.06 \mathrm{eV} /$ atom. The computational supercell consisted of a base metal substrate containing $2048 \mathrm{Cu}$ atoms $(\sim 2.6 \mathrm{~nm} \times 3.6 \mathrm{~nm} \times 2.5 \mathrm{~nm})$ configured with (111) orientation along the surface normal. Periodic boundary conditions were employed in the plane of the substrate (i.e., along $\mathrm{x}$ - and $\mathrm{y}$ - directions). The top 2-3 layers of this $\mathrm{Cu}$ slab $(\sim 0.5-0.6 \mathrm{~nm})$ were first oxidized by exposing it to an oxygen environment in a NVT $\mathrm{MD}$ run at $300 \mathrm{~K}$ for $1 \mathrm{~ns}$. The constant temperature conditions were maintained using the Nosé-Hoover thermostat as implemented in LAMMPS [46]. Representative structures were then chosen from different stages of this oxidation run such that the top oxide layer $\mathrm{CuO}_{x}$ possessed the desired oxygen stoichiometry $x_{O}$. A wide range of values of $x_{O}$ from 0.16 (oxygen deficient) to 0.79 (oxygen enriched) was considered in this study. We note that for cuprous oxide with ideal stoichiometry, i.e., $\mathrm{Cu}_{2} \mathrm{O}$, that value of $x_{O}$ is 0.5 . At each value of $x$, the $\mathrm{Cu}$ slab with surface oxide film was relaxed in vacuum (supercell dimensions: $\sim 2.6 \mathrm{~nm} \times 3.6 \mathrm{~nm} \times 6.5 \mathrm{~nm}$; $4 \mathrm{~nm}$ vacuum normal to the surface) for $100 \mathrm{ps}$ at $300 \mathrm{~K}$ prior to exposing them to aqueous conditions. After thermal relaxation of the 
slab with surface oxide film, the empty space above the slab was filled with $714 \mathrm{H}_{2} \mathrm{O}$, $238 \mathrm{OH}^{-}$ions, and $169 \mathrm{Cl}^{-}$ions (equivalent to $10 \mathrm{M} \mathrm{Cl}^{-}$concentration) at random locations as shown in Figure 1. The number of water molecules was evaluated assuming a density of $1 \mathrm{~g} / \mathrm{cc}$, while the excess hydroxide and $\mathrm{Cl}^{-}$ion concentration was chosen to mimic marine environment such as seawater $[5,6]$. The system was allowed to equilibrate at $300 \mathrm{~K}$ using NVT-MD simulation for $1.5 \mathrm{~ns}$; a subsequent production run of 0.5 ns was used to investigate dynamical and structural properties. It is important to note that in the initial configurations, the charges on all the atoms (including hydroxyl and chloride ions) were set to zero to avoid introduction of any bias. The temporal evolution of the atomic charges was determined via the electronegative equalization method, which is incorporated in the ReaxFF framework. During the MD runs, irrespective of the value of $x_{O}$, the $\mathrm{Cu} / \mathrm{CuO}_{\mathrm{x}}$ slab exhibits a slight positive charge (e.g., $\sim 0.01 \mathrm{e}^{-} /$atom at $x_{O}=0.16$ after $0.1 \mathrm{~ns}$ ), while the aqueous solution possesses a slight negative charge (e.g., $\sim-0.01$ e $/$ atom at $x_{O}=0.16$ after $0.1 \mathrm{~ns}$ ); the overall system, however, remains neutral.

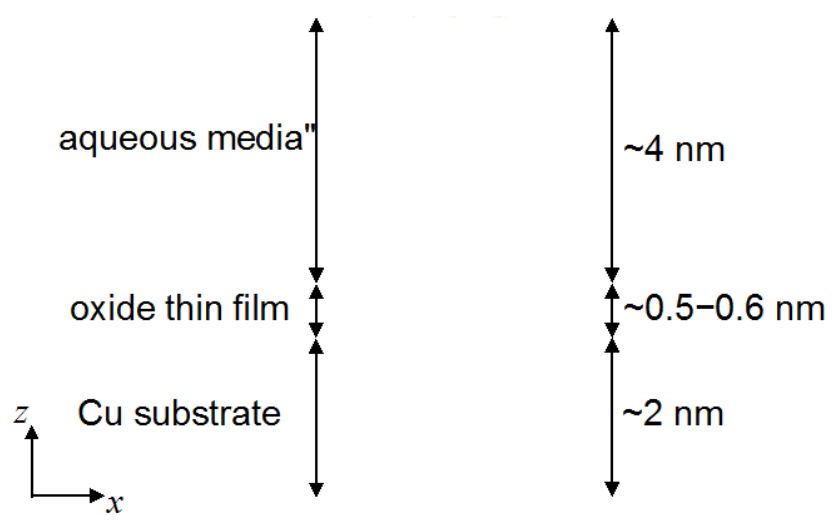

Figure 1: Side view of a typical computational supercell containing a $\mathrm{Cu}$ (111) substrate with an oxide thin film on top being exposed to aqueous media at $10 \mathrm{M} \mathrm{Cl}^{-}$ concentration. The spheres shown here represent copper (tan), oxygen (red), hydrogen (blue), and chlorine (green) atoms. For the sake of clarity, the atoms belonging to aqueous media are represented by smaller spheres. 


\section{Results and Discussion}

\subsection{Effect of stoichiometry on structure and density of passive oxide}

The corrosion behavior of a metal ( $\mathrm{Cu}$ in the present study) in aqueous environment is known to be highly sensitive to the structural condition of the passive surface oxide film formed on top of it $[5,47]$. To characterize the structure of the oxide films prior to exposing them to aqueous media, we evaluated the pair-distribution function (PDF) for $\mathrm{Cu}-\mathrm{O}$ pairs for the surface oxide films for varying oxygen stoichiometry (Fig. 2). The Cu-O PDFs show a characteristic peak at $r=0.185 \mathrm{~nm}-$ $0.190 \mathrm{~nm}$ corresponding to the nearest neighbor distance, which is in excellent agreement with previous X-ray diffraction measurements [48] as well as Monte-Carlo simulations [49]. As expected, the intensity of this nearest-neighbor peak decreases with reduction in $\mathrm{O}$ stoichiometry $x_{O}$ due to the decrease in the number of $\mathrm{O}$ atoms (and in turn, $\mathrm{Cu}-\mathrm{O}$ pairs). At higher $\mathrm{Cu}-\mathrm{O}$ distances, there are no distinct peaks indicating that the $\mathrm{Cu}$-oxide film is amorphous at all values of $x_{O}$. It is interesting to note that the oxide films with higher $\mathrm{O}$ content exhibit slightly better atomic ordering as indicated by a broad peak at $r$

$\sim 0.41 \mathrm{~nm}$ particularly at $x_{O} \geq 0.48$ (Fig. 2). Furthermore, a close-inspection of the nearest-neighbor peaks of the Cu-O PDFs (inset Fig. 2) shows a slight but systematic decrease in the closest $\mathrm{Cu}-\mathrm{O}$ spacing $\left(\sim 5 \times 10^{-3} \mathrm{~nm}\right)$ with increasing $x_{O}$. This is understandable since the thickness of the $\mathrm{Cu}$ oxide films remains nearly constant $\sim 0.5 \mathrm{~nm}$ (Fig. 1) at all values of $x_{O}$, which results in higher atomic packing in O-enriched films. 


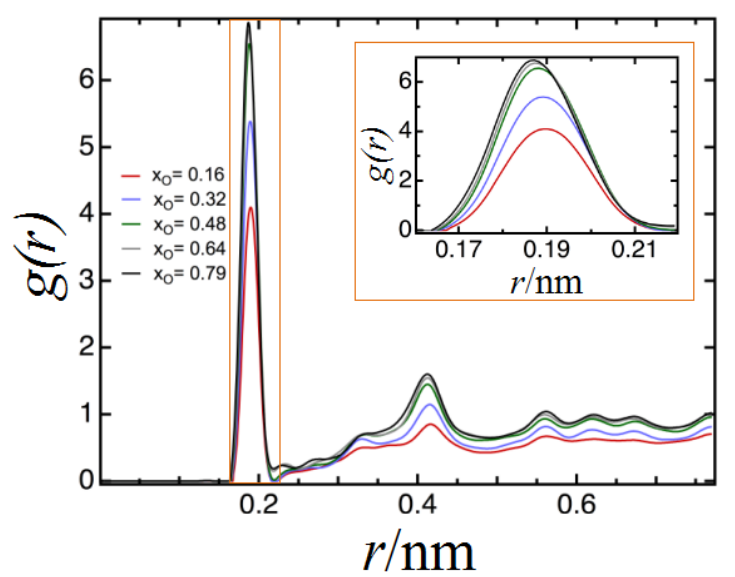

Figure 2: Pair distribution function (PDF) of $\mathrm{Cu}-\mathrm{O}$ pairs in oxide films with different oxygen stoichiometry $x_{0}$. (inset) Magnified view of the nearest neighbor peaks.

3.2 Temporal evolution of passive oxide films with different O-stoichiometry upon chloride ion adsorption

The structural characterization of unexposed Cu-oxide films at different $x_{O}$ indicates that these films are largely amorphous, and possess similar features irrespective of the O-content. This ensures that the aqueous corrosion behavior studied here is governed primarily by the O-content in the surface oxide film rather than any structural differences. In order to assess the corrosion resistance of the $\mathrm{Cu}$-oxide films under aqueous conditions, we evaluated the temporal evolution of the number of chloride ions adsorbed on the oxide films at different $x_{O}$ during our MD runs (Fig. 3(a)). We found that deficiency of $\mathrm{O}$ atoms in the surface oxide layer promotes chloride ion adsorption; in fact, there is a systematic increase in chlorine ion adsorption with decrease in $x_{O}$. For instance, at $x_{O}=0.16$, the number of chloride ions adsorbed are nearly twice as many as that at $x_{O}=0.79$. We also note that most of the chloride ions get adsorbed during the first $\sim 1$ ns, after which, the system is in equilibrium. The adsorbed chloride ions bind with nearby $\mathrm{Cu}$ atoms resulting in the dissociation of existing $\mathrm{Cu}-\mathrm{O}$ bonds, which in turn, leads to chemical thinning of the oxide film. The formation of new $\mathrm{Cu}-\mathrm{Cl}$ bonds also 
manifests itself as a gradual increase (more negative) in the average charge on chloride ion $z_{C l}$ as a function of time (Fig. 3(b)). The temporal evolution of $z_{C l}$ shows that chloride ion charge increases gradually upto $\sim 1 \mathrm{~ns}$, which is consistent with the trend in number of adsorbed chloride ions. Furthermore, as expected from the chloride ion adsorption behavior in Fig. 3(a), the average charge of chloride ions in the non-stoichiometric (low $x_{O}$ ) cases are higher (as high as $\sim 20 \%$ ) as compared to the more stoichiometric (high $x_{O}$ ) ones.
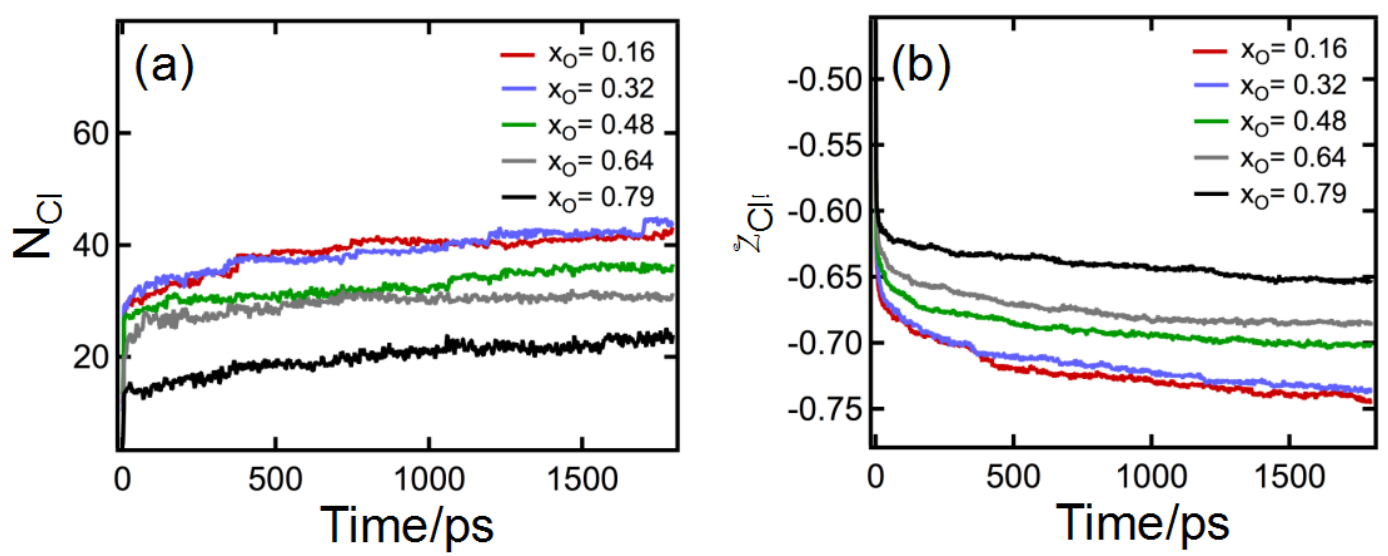

Figure 3: Time evolution of chloride ion adsorption on passive oxide films with different $\mathrm{O}$-stoichiometry (a) number of adsorbed chloride ions, (b) average charge on chloride ions.

\subsection{Effect of surface oxide stoichiometry on chloride ion adsorption}

Figure 4 shows the snapshots of the top view of Cu-oxide films with different Ostoichiometry at different times $t$ during our MD simulations of aqueous corrosion. Upon exposing the oxide films to aqueous media, chloride ions begin to adsorb on to the surface; consistent with Fig. 3, we observe that higher number of chloride ions adsorb on the oxide films with low O-content, and distinctly thin the passive oxide film. Chloride ions adsorbed on the oxide film facilitate the dissolution of $\mathrm{Cu}$ atoms from the oxide film into the aqueous media via dissociation of existing $\mathrm{Cu}-\mathrm{O}$ bonds, similar to our previous 
findings [5]. Furthermore, we found that an adsorbed chloride ion promotes further adsorption leading to formation of a wide range of soluble $\mathrm{Cu}-\mathrm{Cl}$ complexes; these complexes subsequently dissolve into the aqueous medium. These direct observations clearly indicate that higher chloride ion adsorption on the passive oxide surface eventually causes higher corrosion of underlying Cu substrate. Our findings are in good agreement with the mechanism of pitting corrosion proposed earlier based on potentiostatic polarization and X-ray photoelectron spectroscopy, which suggested that dissolution of $\mathrm{Cu}$ atoms must occur via soluble complexes like $\mathrm{CuCl}_{2}{ }^{-}[50]$.

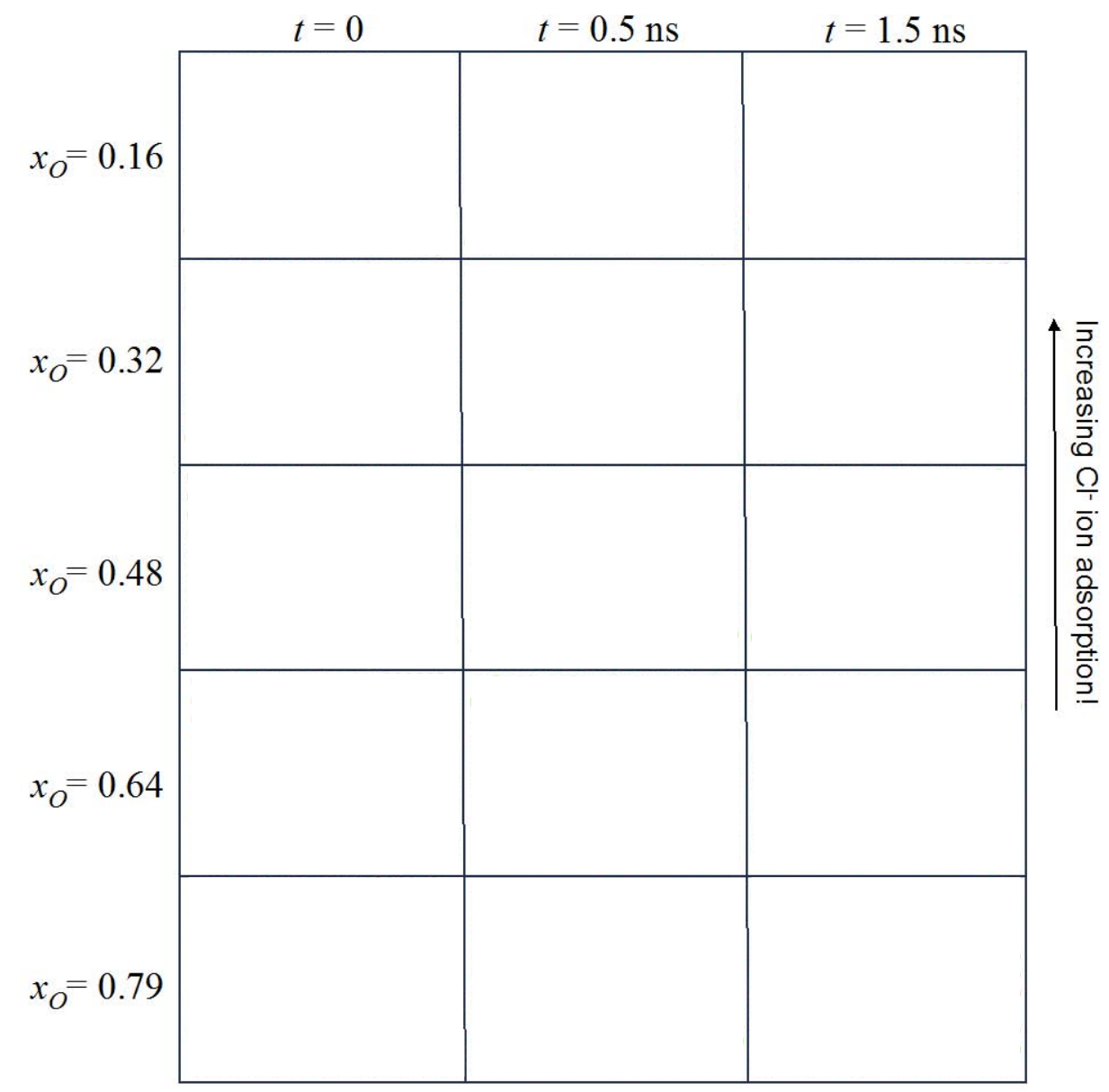

Figure 4: Top view of the interface between the passive oxide layer and aqueous medium at selected times during our MD simulations of corrosion. For the sake of clarity, the adsorbed chloride ions are shown as big spheres (green), while the copper (tan), oxygen (red), and hydrogen (blue) atoms are shown are shown are small spheres. 
To further investigate the extent of dissolution of $\mathrm{Cu}$ atoms as well as adsorption of $\mathrm{Cl}$ ions, we computed the atomic number density distribution of $\mathrm{Cl}\left(\rho_{\mathrm{Cl}}\right)$ and $\mathrm{Cu}\left(\rho_{\mathrm{Cu}}\right)$ atoms along the surface normal direction (z-axis) as a function of the O-stoichiometry in the oxide film (Fig. 5). The density distributions were averaged over the configuration obtained in the last 0.5 ns of our aqueous corrosion MD simulations. As shown in Fig. 1, the interface between the passive oxide film and the aqueous media occurs at $z \sim 2.5 \mathrm{~nm}$. Proximal to this interface, we find an increase in the density of $\mathrm{Cl}$ atoms providing evidence for chloride ion adsorption. Consistent with the findings in Fig. 2(a), we find that $\mathrm{Cl}$ adsorption is the highest on the oxide films with very low oxygen content $\left(x_{O}=\right.$ 0.2 ) as indicated by highest intensity of the $\rho_{C l}$ peak at $\sim 2.6 \mathrm{~nm}$ in Fig. 5(a). As the Ocontent increases, the amount of $\mathrm{Cl}$ adsorption drops significantly as illustrated by the drastic decrease in the intensity of $\rho_{C l}$ at the oxide-aqueous media interface. It is also important to note that there is considerable infiltration of chloride ions into the oxide layer, particularly at low values of $x_{O}$ as shown by peaks of medium intensity; e.g., $\rho_{C l}$ increases to $\sim 1.5 \times 10^{-5} \mathrm{~nm}^{-3}$ in the top layer of oxide film at $x_{O}=0.2$.
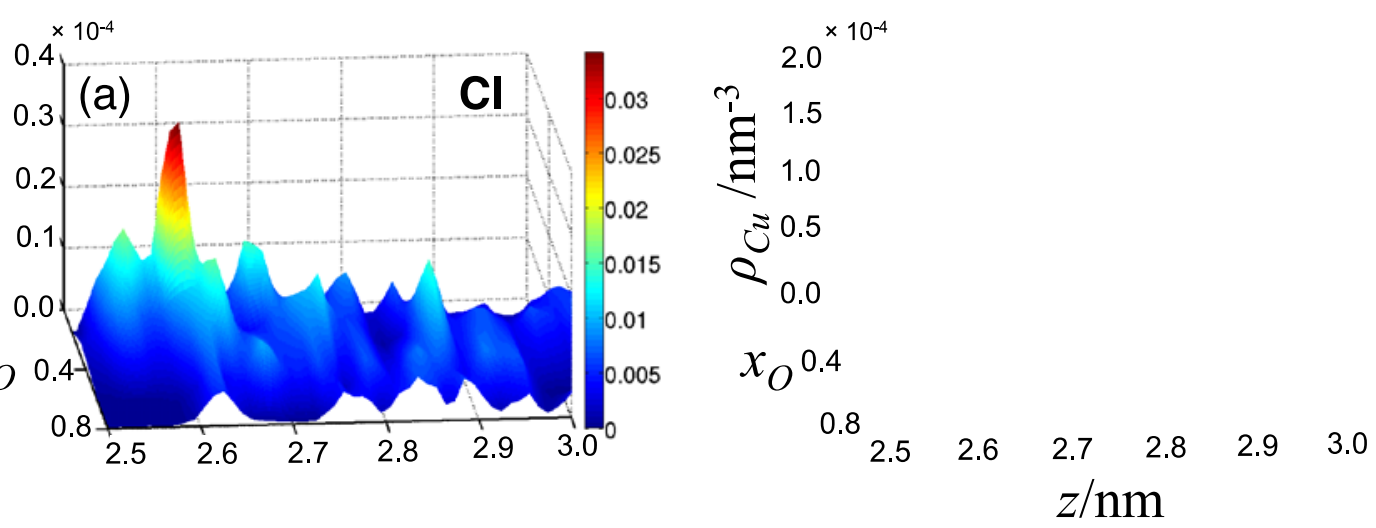

Figure 5: Effect of oxygen stoichiometry on the atomic density profile $\rho$ of (a) $\mathrm{Cl}$, and (b) $\mathrm{Cu}$ in a direction normal to the oxide-aqueous media interface. For the sake 
of clarity, only the spatial regions close to the oxide-aqueous media interface $(\mathrm{z} \sim 2.5-2.6 \mathrm{~nm})$ are shown.

In terms of removal of $\mathrm{Cu}$ atoms from the oxide surface, most $\mathrm{Cu}$ dissolution takes place from oxide films of low O-content (e.g., see $\rho_{\text {Cu }}$ peak at $z \sim 2.65 \mathrm{~nm}$ at $x_{O}=$ 0.2 in Fig. 5(b)). Furthermore, the smearing of the $\rho_{C u}$ peak at the oxide-aqueous media interface reveals that $\mathrm{Cu}$ atoms removed from O-deficient films dissolve further into the aqueous media, and results in significant thinning of the oxide film. To better understand the spatial arrangement of $\mathrm{Cu}$ atoms around the adsorbed chloride ions, we computed the PDFs of $\mathrm{Cu}-\mathrm{Cl}$ pairs as a function of O-stoichiometry (Fig. 6). These PDFs show that the nearest neighbor spacing between $\mathrm{Cu}$ and $\mathrm{Cl}$ atoms is $0.22 \mathrm{~nm}-0.235 \mathrm{~nm}$; these values are in agreement with previous x-ray diffraction studies on aqueous $\mathrm{CuCl}_{2}$, which report the $\mathrm{Cu}-\mathrm{Cl}$ bond length to be $\sim 0.225-0.243 \mathrm{~nm}[48,51]$. By analyzing the coordination of $\mathrm{Cl}$ around $\mathrm{Cu}$ atoms within the predicted nearest neighbor distance $(r \sim 0.22-0.235$ $\mathrm{nm}$ ), we found that the $\mathrm{Cu}-\mathrm{Cl}$ pairs can exists as $\mathrm{CuCl}, \mathrm{CuCl}_{2}$ or $\mathrm{CuCl}_{3}$, especially at low values of $x_{O}=0.2$. This is in good agreement with previous $\mathrm{MD}$ simulations of $\mathrm{Cu}$ corrosion [5] as well as x-ray photoelectron spectroscopy studies, which report the formation of $\mathrm{CuCl}_{2}^{-}$ions during the corrosion of $\mathrm{Cu}$ in chloride solutions [50]. In Fig. 6, it is also interesting to note that the intensity of the nearest-neighbor peak in the PDF of $\mathrm{Cu}-\mathrm{Cl}$ decreases with increase in the O-content of the oxide films. This provides further evidence for higher chloride adsorption, and consequent higher thinning of the oxide films with low values of $x_{O}$. 


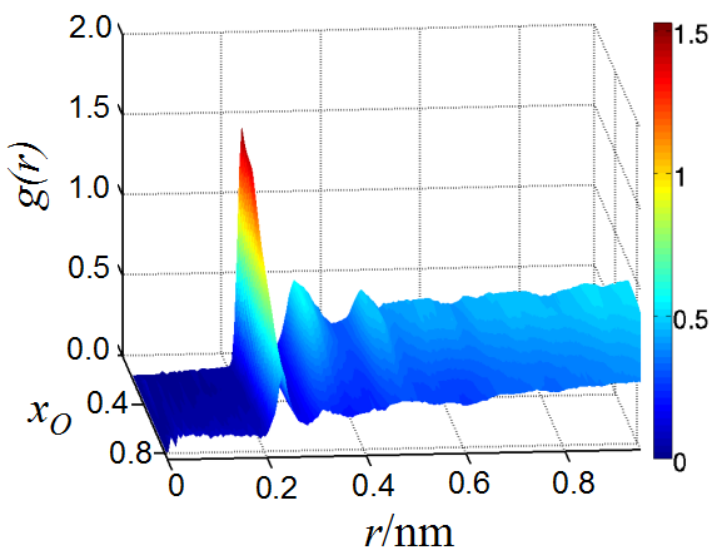

Figure 6: Effect of oxygen stoichiometry on pair distribution function for $\mathrm{Cu}-\mathrm{Cl}$ pairs averaged over the last $0.5 \mathrm{~ns}$ of $\mathrm{MD}$ simulations

3.4 Effect of surface oxide stoichiometry on solvation dynamics and molecular ordering of water

The above results clearly suggest that the oxide non-stoichiometry has a pronounced effect on the local density fluctuations and adsorption of the chloride ions. In this section, we further evaluate the impact of non-stoichiometry on the structure and dynamics of water layers in the vicinity of an oxide surface.

3.4.1 Residence probability of interfacial layers as a function of passive oxide nonstoichiometry

We have calculated the residence time probability $\left(P_{\text {res }}(t)\right)$ of interfacial water molecules as a function of the oxide stoichiometry. To facilitate comparisons between the various cases, the water molecules were divided into two different regions: (1) interfacial water molecules (water molecules located in the vicinity of the passive oxide layer i.e. within $1.5 \mathrm{~nm}$ ) (2) bulk water (water molecules located beyond $1.5 \mathrm{~nm}$ from the oxide surface). $P_{\text {res }}(t)$ was defined in both the cases, as the probability of finding a water molecule in one of the above defined regions without departing from that region for the time interval $t_{0}$ and $t+t_{0}[52,53]$. $P_{\text {res }}(t)$ was averaged over several different simulation frames that were collected over the total time domain of 1 ns with intervals of 1 ps. 
Figure 7 shows the $P_{\text {res }}(t)$ for interfacial water molecules as a function of the varying oxygen stoichiometry of the passive oxide surface. These calculations allowed us to quantify on an average, how long, a particular water molecule continuously resides in any given region, namely, either interfacial or bulk water. We find that $P_{\text {res }}(t)$ of interfacial water molecules decreases with increase in the extent of non-stoichiometry [Fig. 7]. Water molecules reside for longer time in the case of near-stoichiometric surface $\left(x_{O}>0.7\right)$ as compared to water molecules for non-stoichiometric oxides $\left(x_{O}<0.5\right)$. The slow dynamical behavior for interfacial water near stoichiometric oxides is suggestive of an ordered arrangement of water molecules, which is also an energetically stable configuration. The timescale for the residence time $P_{\text {res }}(t)$ to decay to $1 / e$ is $>>1$ ns for interfacial water molecules corresponding to $\left(x_{O}>0.5\right)$ whereas $P_{\text {res }}(t)$ decays ca. 100200 ps for $x_{O}<0.5$. Note that the $P_{\text {res }}(t)$ for bulk water is $\sim 8$ ps which is similar to that obtained by Argyris et al. [54] As the extent of oxygen non-stoichiometry is increased, the dynamical properties of the interfacial water molecules begin to approach the properties shown by bulk water whereas in the near stoichiometric case $\left(x_{O} \sim 0.8\right)$, the interfacial layers dynamically change and relax to energetically stable configurations of ordered water. It is worth noting that $P_{\text {res }}(t)$ obtained using the intermittent definition also showed a similar trend. 


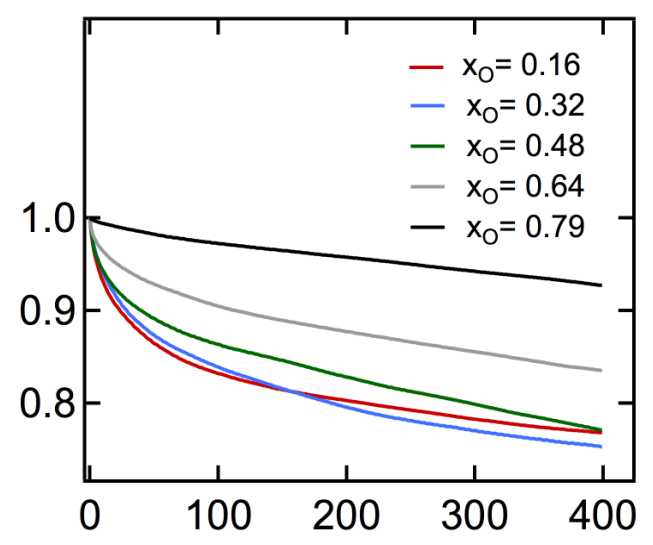

Figure 7: Effect of passive oxide non-stoichiometry on the residence probability of water molecules on $\mathrm{Cu}$-oxide surface.

\subsubsection{Hydrogen bond correlation function of interfacial layers as a function of passive oxide non-stoichiometry}

To further explore ordering of water at the metal-oxide/aqueous medium interface, we performed HB analysis by employing a well-known configurational criterion [55]. In short, two water molecules are considered to be connected via HBs only if the oxygen atoms of the two water molecules are separated by a distance $<0.35 \mathrm{~nm}$, as well as the $\mathrm{O}-\mathrm{H} \ldots \mathrm{O}$ angle is less than $30^{\circ}$ following our earlier works $[31,56]$. To identify whether a pair of water molecules are hydrogen bonded, we use a binary function $h(t)$ following Ref. [55]. The value of $h$ is unity when the tagged pair is hydrogen bonded, and is zero otherwise. In the dynamical equilibrium of liquid water, the fluctuations in $h$ are characterized by the correlation function [55].

$$
C_{H B}(t)=\frac{\langle h(0) h(t)\rangle}{\left\langle h^{2}\right\rangle}
$$

where $h(t)$ refers to the binary function for every pair of water molecules at time $t$, and the angle brackets denote average over all distinct pairs of water molecules and initial timeframes. Furthermore, HB lifetime $\tau_{H B}$ is defined such that $C_{H B}\left(\tau_{H B}\right)=e^{-1}$. 
We calculate the HB correlation function and lifetimes for the interfacial water layers as a function of the passive oxide non-stoichiometry. We find that our calculated HB lifetimes comply with the diffusivity variation. In bulk water, it should be noted that the $\tau_{H B}$ are $\sim 5-6$ ps which is reminiscent of the intermittent collective motion of water molecules as a result of extensive HB network rearrangement dynamics of liquid water [57]. In the case of interfacial water near a more stoichiometric oxide, HB correlation function decays much slower with lifetimes of $\sim 250 \mathrm{ps}$, which implies characteristics of 2-D layered water (Fig. 8). A slow dynamical behavior indicates energetically stable configurations of interfacial water wherein significant extent of ordering is still preserved. This is consistent with previously reported simulations of Balasubramanian et al. [58] and our recent simulations on magnesium oxide-aqueous interfaces [18]. They observed that both the residence times and $\tau_{\mathrm{HB}}$ among interfacial water molecules are much higher than bulk owing to the ordering.

As the extent of non-stoichiometry is increased from 0.79 to 0.2 , we observe a significant lowering in the HB lifetimes; the corresponding HB correlation shows a much faster decay and approaches that of bulk water. This suggests that the interfacial water layers in the case of highly non-stoichiometric oxides are much more disordered than those that are nearly stoichiometric. The observed variation in the $\tau_{\mathrm{HB}}$ can be explained by the fact that interfacial water molecules are strongly hydrogen-bonded to the passive oxide surface and hence have much higher $\tau_{H B}$ than pure bulk water. With an increase in oxygen non-stoichiometry, we find that the HB correlation function decays slightly faster, leading to reduced $\tau_{\mathrm{HB}}$. A strong decrease is seen when $x_{O}<0.5$, which 
coincides with the disruption of the ordered interfacial water. The $\tau_{H B}$ at these high levels of non-stoichiometry are $180-1420$ ps.

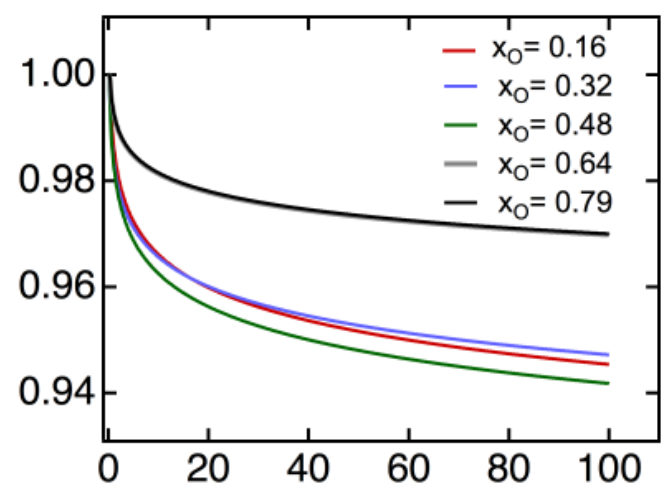

Figure 8: Hydrogen bond correlation function for the interfacial water layers as a function of the passive oxide non-stoichiometry.

\subsubsection{Diffusivity of water molecules and chloride ions}

We then consider the relationship between the molecular order and transport characteristics of water and halide ions. We show in Fig. 9(a) and (b) the calculated dependence of the diffusion coefficient $D$ of chloride ions and water molecules, respectively as a function of the non-stoichiometry of the passive oxide. Our reactive MD simulations suggest atomic diffusivity of interfacial water depends strongly on the extent of non-stoichiometry. In particular, we find that diffusion coefficients of water near a more stoichiometric oxide are fairly small $\left(D \sim 2 \times 10^{-5} \mathrm{~cm}^{2} / \mathrm{s}\right.$ for $x_{O} \sim 0.8$ ), comparable to those in a copper oxide without any $\mathrm{Cl}$ - ions. As the extent of nonstoichiometry increases, however, we observe that the diffusion of water becomes significant ( $D \sim 16 \times 10^{-5} \mathrm{~cm}^{2} / \mathrm{s}$ for $\left.x_{O} \sim 0.2\right)$. There is a facile rearrangement of atoms in the layered structure at such higher non-stoichiometry levels. Closer analysis of the MD trajectories suggests significant disruption of the quasi-two dimensional interfacial water 
structure. It should be noted that there is a significant loss of molecular order as a result of rapid diffusion of the chloride ions; their diffusivity increases from $0.2 \times 10^{-5} \mathrm{~cm}^{2} / \mathrm{s}$ to $1.4 \times 10^{-5} \mathrm{~cm}^{2} / \mathrm{s}$ as oxygen stoichiometry changes from $x_{O} \sim 0.80$ to $x_{O} \sim 0.20$. This, in turn, suggests that rapid adsorption of chloride ions to the passive oxide should be possible for high levels of oxide non-stoichiometry. Indeed, this behavior is corroborated by the atomic density profile of $\mathrm{Cl}$ ions shown in Fig. 5(a).

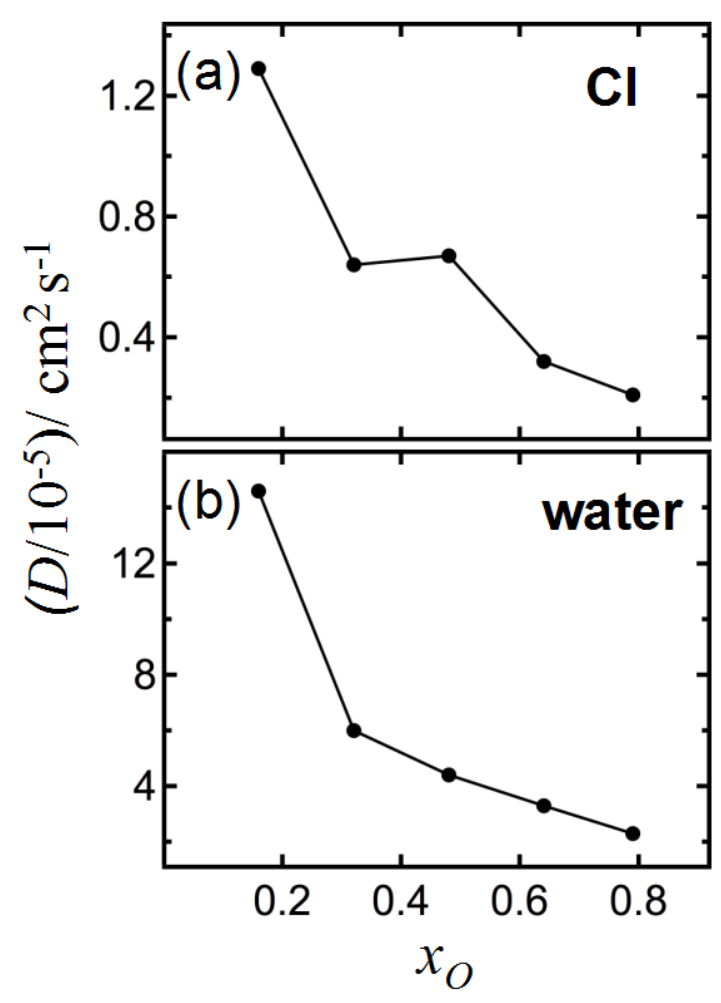

Figure 9: Diffusion coefficients of (a) chloride ions and (b) water molecules at room temperature as a function of the passive oxide stoichiometry.

The observed effect of oxide non-stoichiometry on the molecular order, structure and dynamics of aqueous interfaces is rather interesting. In a typical ordered aqueous interface such ice-water system, there is a tendency for chloride ion rejection from the ordered ice phase into water (phenomenon is termed brine rejection). The increased propensity of water to solvate chloride ions leads to their expulsion from an 
ordered ice structure into liquid water as demonstrated in the MD simulations by Jungwirth and co-workers [59]. The ordered quasi-two dimensional structure near the passive oxide is therefore expected to suppress corrosion by having a similar tendency to expel chloride ions and maintain its ordered structure as in the ice-water case.

We, however, observe that the extent of interfacial ordering is a strong function of the oxide stoichiometry. We note that the extent of non-stoichiometry can lead to an imbalance in the distribution of the charges; the average surface charges are higher when the extent of non-stoichiometry is higher. For higher levels of oxide non-stoichiometry, the surface preference of the chloride ions due to long-range Coulombic interactions seems to overcome the tendency to expel these ions into the bulk of the liquid.

\section{Conclusions}

MD simulations indicate that breakdown of the passive Cu-oxide films proceeds by adsorption of $\mathrm{Cl}^{-}$ions onto the film (from the aqueous medium), which preferentially bind to $\mathrm{Cu}$ atoms in the oxide and form soluble $\mathrm{Cu}-\mathrm{Cl}$ complexes. These complexes dissolve in the aqueous medium resulting in $\mathrm{Cu}$ dissolution and chemical thinning of the film. We explored the role of interfacial solvation dynamics in influencing the dissolution of $\mathrm{Cu}$. In the close proximity of the O-rich oxide films, we found that water molecules arrange in well-ordered quasi-2D layers as indicated by high residence probabilities and hydrogen bond lifetimes. These layers are energetically favorable, as evidenced by the slow diffusion characteristics of the water molecules belonging to these layers. For a near stoichiometric oxide, the two-dimensional ordered layers of water (reminiscent of icelike) tend to expel the chloride ions back into the disordered regions far away from the oxide/aqueous medium interface. At higher oxygen non-stoichiometry, we observe 
chloride ion induced disruption of these ordered layers, which facilitates aggressive dissolution and breakdown of passive oxide film. Our study indicates that reduced $\mathrm{Cl}^{-}$ ion adsorption occurs not only due to impaired Coulomb interactions owing to the presence of low surface charges in O-rich films, but also due to interesting solvation effects at the metal-oxide/aqueous medium interface.

\section{Acknowledgements}

Use of the Center for Nanoscale Materials was supported by the U. S. Department of Energy, Office of Science, Office of Basic Energy Sciences, under Contract No. DEAC02-06CH11357. This research used resources of the National Energy Research

Scientific Computing Center, a DOE Office of Science User Facility supported by the Office of Science of the U.S. Department of Energy under Contract No. DE-AC0205CH11231. An award of computer time was provided by the Innovative and Novel Computational Impact on Theory and Experiment (INCITE) program. This research used resources of the Argonne Leadership Computing Facility, which is a DOE Office of Science User Facility supported under Contract DE-AC02-06CH11357.

\section{References:}

[1] C. Punckt, M. Bolscher, H.H. Rotermund, A.S. Mikhailov, L. Organ, N. Budiansky, J.R. Scully, J.L. Hudson. Sudden onset of pitting corrosion on stainless steel as a critical phenomenon, Science 305 (2004) 1133-1136.

[2] H.J. Bakker. Water dynamics ion-ing out the details, Nature Chemistry 1 (2009) 24-25.

[3] F.U. Renner, A. Stierle, H. Dosch, D.M. Kolb, T.L. Lee, J. Zegenhagen. Initial corrosion observed on the atomic scale, Nature 439 (2006) 707-710.

[4] C. Barth, M. Reichling. Imaging the atomic arrangements on the high-temperature reconstructed alpha-Al2O3(0001) surface, Nature 414 (2001) 54-57. 
[5] B. Jeon, S.K.R.S. Sankaranarayanan, A.C.T. van Duin, S. Ramanathan. Reactive Molecular Dynamics Study of Chloride Ion Interaction with Copper Oxide Surfaces in Aqueous Media, Acs Applied Materials \& Interfaces 4 (2012) 1225-1232.

[6] J. Byoungseon, S.K.R.S. Sankaranarayanan, A.C.T. van Duin, S. Ramanathan. Atomistic insights into aqueous corrosion of copper, Journal of Chemical Physics 134 (2011) 234706 (234710 pp.)-234706 (234710 pp.).

[7] F. Bruni, S. Imberti, R. Mancinelli, M.A. Ricci. Aqueous solutions of divalent chlorides: Ions hydration shell and water structure, Journal of Chemical Physics 136 (2012).

[8] G.H. Gilmer. Computer-models of crystal-growth, Science 208 (1980) 355-363.

[9] B. Jeon, S.K.R.S. Sankaranarayanan, A.C.T. van Duin, S. Ramanathan. Influence of surface orientation and defects on early-stage oxidation and ultrathin oxide growth on pure copper, Philosophical Magazine 91 (2011) 4073-4088.

[10] P. Marcus, J.M. Herbelin. The entry of chloride-ions into passive films on nickel studied by spectroscopic (esca) and nuclear (cl-36 radiotracer) methods, Corrosion Science 34 (1993) 1123-1145.

[11] J.A. Bardwell, B. Macdougall, G.I. Sproule. Use Of Sims To Investigate The Induction Stage In The Pitting Of Iron, Journal of the Electrochemical Society 136 (1989) 1331-1336.

[12] D.A. Little, M.A. Jakab, J.R. Scully. Effect of surface pretreatment on the underpaint corrosion of AA2024-T3 at various temperatures, Corrosion 62 (2006) 300-

315.

[13] L.J. Criscenti, R.T. Cygan, A.S. Kooser, H.K. Moffat. Water and halide adsorption to corrosion surfaces: Molecular simulations of atmospheric interactions with aluminum oxyhydroxide and gold, Chemistry of Materials 20 (2008) 4682-4693.

[14] C. Stampfl, M.V. Ganduglia-Pirovano, K. Reuter, M. Scheffler. Catalysis and corrosion: the theoretical surface-science context, Surface Science 500 (2002) 368-394. [15] L.F. Lin, C.Y. Chao, D.D. Macdonald. A point-defect model for anodic passive films .2. Chemical breakdown and pit initiation, Journal of the Electrochemical Society 128 (1981) 1194-1198.

[16] C.Y. Chao, L.F. Lin, D.D. Macdonald. A point-defect model for anodic passive films .1. Film growth-kinetics, Journal of the Electrochemical Society 128 (1981) 11871194.

[17] D.D. Macdonald. The point-defect model for the passive state, Journal of the Electrochemical Society 139 (1992) 3434-3449.

[18] S. Deshmukh, G. Kamath, S. Ramanathan, S.K.R.S. Sankaranarayanan. Chloride ions induce order-disorder transition at water-oxide interfaces, Physical Review E 88 (2013) 062119.

[19] I.A. Suleiman, M.W. Radny, M.J. Gladys, P.V. Smith, J.C. Mackie, E.M.

Kennedy, B.Z. Dlugogorski. Chlorination of the Cu(110) Surface and Copper

Nanoparticles: A Density Functional Theory Study, Journal of Physical Chemistry C 115 (2011) 13412-13419.

[20] I.A. Suleiman, M.W. Radny, M.J. Gladys, P.V. Smith, J.C. Mackie, E.M.

Kennedy, B.Z. Dlugogorski. An equilibrium ab initio atomistic thermodynamics study of chlorine adsorption on the $\mathrm{Cu}(001)$ surface, Physical Chemistry Chemical Physics 13 (2011) 10306-10311. 
[21] M. Altarawneh, M.W. Radny, P.V. Smith, J.C. Mackie, E.M. Kennedy, B.Z. Dlugogorski, A. Soon, C. Stampfl. A first-principles density functional study of chlorophenol adsorption on Cu2O(110):CuO, Journal of Chemical Physics 130 (2009). [22] Y.S. Chu, T.E. Lister, W.G. Cullen, H. You, Z. Nagy. Commensurate water monolayer at the RuO2(110)/water interface, Physical Review Letters 86 (2001) 33643367.

[23] L. Cheng, P. Fenter, K.L. Nagy, M.L. Schlegel, N.C. Sturchio. Molecular-scale density oscillations in water adjacent to a mica surface, Physical Review Letters 87 (2001).

[24] P. Fenter, L. Cheng, C. Park, Z. Zhang, N.C. Sturchio. Structure of the orthoclase (001)- and (010)-water interfaces by high-resolution X-ray reflectivity, Geochimica Et Cosmochimica Acta 67 (2003) 4267-4275.

[25] P. Fenter, H. Teng, P. Geissbuhler, J.M. Hanchar, K.L. Nagy, N.C. Sturchio. Atomic-scale structure of the orthoclase (001)-water interface measured with highresolution X-ray reflectivity, Geochimica Et Cosmochimica Acta 64 (2000) 3663-3673. [26] M.F. Toney, J.N. Howard, J. Richer, G.L. Borges, J.G. Gordon, O.R. Melroy, D.G. Wiesler, D. Yee, L.B. Sorensen. Voltage-dependent ordering of water-molecules at an electrode-electrolyte interface, Nature 368 (1994) 444-446.

[27] P. Fenter, N.C. Sturchio. Mineral-water interfacial structures revealed by synchrotron X-ray scattering, Progress in Surface Science 77 (2004) 171-258.

[28] S.A. Deshmukh, S.K.R.S. Sankaranarayanan. Atomic scale characterization of interfacial water near an oxide surface using molecular dynamics simulations, Physical Chemistry Chemical Physics 14 (2012) 15593-15605.

[29] G. Kamath, S.A. Deshmukh, S.K.R.S. Sankaranarayanan. Comparison of select polarizable and non-polarizable water models in predicting solvation dynamics of water confined between MgO slabs, Journal of Physics-Condensed Matter 25 (2013).

[30] M. Odelius, M. Bernasconi, M. Parrinello. Two dimensional ice adsorbed on mica surface, Physical Review Letters 78 (1997) 2855-2858.

[31] S. Deshmukh, G. Kamath, S.K.R.S. Sankaranarayanan. Effect of Nanoscale Confinement on Freezing of Modified Water at Room Temperature and Ambient Pressure, Chemphyschem 15 (2014) 1632-1642.

[32] P. Sung-Ho, G. Sposito. Structure of water adsorbed on a mica surface, Physical Review Letters 89 (2002) 085501-085501-085503.

[33] S. Park, D.E. Moilanen, M.D. Fayer. Water dynamics - The effects of ions and nanoconfinement, Journal of Physical Chemistry B 112 (2008) 5279-5290.

[34] D.E. Moilanen, D. Wong, D.E. Rosenfeld, E.E. Fenn, M.D. Fayer. Ion-water hydrogen-bond switching observed with 2D IR vibrational echo chemical exchange spectroscopy, Proceedings of the National Academy of Sciences of the United States of America 106 (2009) 375-380.

[35] A.C.T. van Duin, S. Dasgupta, F. Lorant, W.A. Goddard. ReaxFF: A reactive force field for hydrocarbons, Journal of Physical Chemistry A 105 (2001) 9396-9409. [36] A.C.T. van Duin, A. Strachan, S. Stewman, Q.S. Zhang, X. Xu, W.A. Goddard. ReaxFF(SiO) reactive force field for silicon and silicon oxide systems, Journal of Physical Chemistry A 107 (2003) 3803-3811. 
[37] K. Chenoweth, A.C.T. van Duin, W.A. Goddard. ReaxFF reactive force field for molecular dynamics simulations of hydrocarbon oxidation, Journal of Physical Chemistry A 112 (2008) 1040-1053.

[38] O. Rahaman, A.C.T. van Duin, V.S. Bryantsev, J.E. Mueller, S.D. Solares, W.A. Goddard, III, D.J. Doren. Development of a ReaxFF Reactive Force Field for Aqueous Chloride and Copper Chloride, Journal of Physical Chemistry A 114 (2010) 3556-3568.

[39] A.K. Rappe, W.A. Goddard. Charge equilibration for molecular-dynamics simulations, Journal of Physical Chemistry 95 (1991) 3358-3363.

[40] J.A. Keith, D. Fantauzzi, T. Jacob, A.C.T. van Duin. Reactive forcefield for simulating gold surfaces and nanoparticles, Physical Review B 81 (2010).

[41] A.C.T. van Duin, V.S. Bryantsev, M.S. Diallo, W.A. Goddard, O. Rahaman, D.J. Doren, D. Raymand, K. Hermansson. Development and Validation of a ReaxFF Reactive Force Field for $\mathrm{Cu}$ Cation/Water Interactions and Copper Metal/Metal Oxide/Metal Hydroxide Condensed Phases, Journal of Physical Chemistry A 114 (2010) 9507-9514. [42] Q. Zhang, T. Cagin, A. van Duin, W.A. Goddard, Y. Qi, L.G. Hector. Adhesion and nonwetting-wetting transition in the Al/alpha-Al2O3 interface, Physical Review B 69 (2004).

[43] D. Raymand, A.C.T. van Duin, M. Baudin, K. Hermansson. A reactive force field (ReaxFF) for zinc oxide, Surface Science 602 (2008) 1020-1031.

[44] W.A. Goddard, Q.S. Zhang, M. Uludogan, A. Strachan, T. Cagin. The ReaxFF polarizable reactive force fields for molecular dynamics simulation of ferroelectrics. Fundamental Physics of Ferroelectrics 2002 Workshop, vol. 626. Washington, D.C., 2002. p.45-55.

[45] B. Narayanan, A.C.T. van Duin, B.B. Kappes, I.E. Reimanis, C.V. Ciobanu. A reactive force field for lithium-aluminum silicates with applications to eucryptite phases, Modelling and Simulation in Materials Science and Engineering 20 (2012).

[46] S. Plimpton. Fast parallel algorithms for short-range molecular-dynamics, Journal of Computational Physics 117 (1995) 1-19.

[47] B. Bernard. Further Insights on the Pitting Corrosion of Stainless Steels. Corrosion Mechanisms in Theory and Practice. CRC Press, 2002. pp. 311-347.

[48] J.R. Bell, J.L. Tyvoll, D.L. Wertz. Solute structuring in aqueous copper(II) chloride solutions, Journal of the American Chemical Society 95 (1973) 1456-1459.

[49] N.R. Texler, B.M. Rode. Monte Carlo simulations of copper chloride solutions at various concentrations including full 3-body correction terms, Chemical Physics 222 (1997) 281-288.

[50] A. El Warraky, H.A. El Shayeb, E.M. Sherif. Pitting corrosion of copper in chloride solutions, Anti-Corrosion Methods and Materials 51 (2004) 52-61.

[51] M. Magini. Hydration and complex-formation study on concentrated-solutions $\mathrm{Co}(11) \mathrm{Cl} 2 \mathrm{Ni}(11) \mathrm{Cl} 2 \mathrm{Cu}(11) \mathrm{Cl} 2$ by x-ray-diffraction technique, Journal of Chemical Physics 74 (1981) 2523-2529.

[52] S.A. Deshmukh, G. Kamath, S.K.R.S. Sankaranarayanan. Comparison of the interfacial dynamics of water sandwiched between static and free-standing fully flexible graphene sheets, Soft Matter 10 (2014) 4067-4083.

[53] S.A. Deshmukh, G. Kamath, G.A. Baker, A.V. Sumant, S.K.R.S. Sankaranarayanan. The interfacial dynamics of water sandwiched between graphene sheets are governed by the slit width, Surface Science 609 (2013) 129-139. 
[54] D. Argyris, N.R. Tummala, A. Strioli, D. Cole. Molecular Structure and Dynamics in Thin Water Films at the Silica and Graphite Surfaces, J. Phys. Chem. C 112 (2008) 13587-13599.

[55] F.W. Starr, J.K. Nielsen, H.E. Stanley. Fast and slow dynamics of hydrogen bonds in liquid water, Physical Review Letters 82 (1999) 2294-2297.

[56] S.A. Deshmukh, S.K.R.S. Sankaranarayanan, K. Suthar, D.C. Mancini. Role of Solvation Dynamics and Local Ordering of Water in Inducing Conformational Transitions in Poly(N-isopropylacrylamide) Oligomers through the LCST, Journal of Physical Chemistry B 116 (2012) 2651-2663.

[57] M. Matsumoto, S. Saito, I. Ohmine. Nature 416 (2002) 409-413.

[58] S. Balasubramanian, S. Pal, B. Bagchi. Hydrogen-bond dynamics near a micellar surface: Origin of the universal slow relaxation at complex aqueous interfaces, Physical Review Letters 89 (2002).

[59] L. Vrbka, P. Jungwirth. Brine rejection from freezing salt solutions: A molecular dynamics study, Physical Review Letters 95 (2005). 


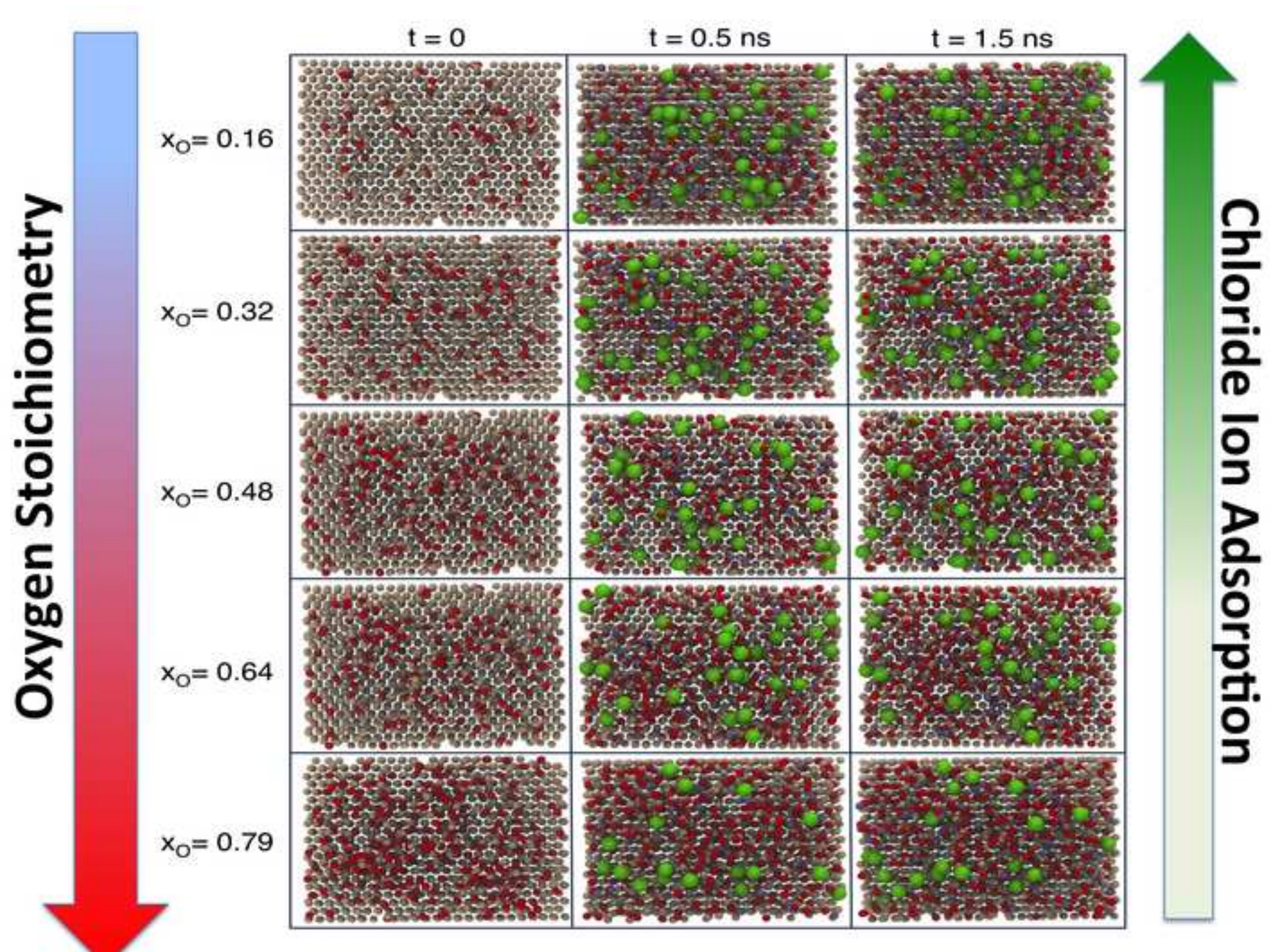

